\title{
Percutaneous transfemoral approach and additional vascular access selection influence hospital stay and survival after transcatheter aortic valve implantation
}

\author{
DIrzal Hadžibegović* , \\ DDanijel Unić, \\ (DIvana Jurin, \\ Nikola Bradić, \\ DBoris Starčević, \\ DIgor Rudež
}

University Hospital Dubrava, Zagreb, Croatia

RECEIVED:

December 6, 2020

ACCEPTED:

December 18, 2020

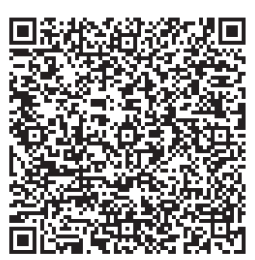

$\square$ Cardiologia Croatica 2021;16(1-2):36.
KEYWORDS: transcatheter aortic valve implantation, vascular access, complications.
CITATION: Cardiol Croat. 2021:16(1-2):36. I https://doi.org/10.15836/ccar2021.36

*ADDRESS FOR CORRESPONDENCE: Irzal Hadžibegović, Klinička bolnica Dubrava, Avenija Gojka Šuška 6, HR-10000 Zagreb, Croatia. / Phone: +385-91-5333091 / E-mail: irzalh@gmail.com

ORCID: Irzal Hadžibegović, https://orcid.org/0000-0002-3768-9134 • Danijel Unić, https://orcid.org/0000-0003-2740-4067 Ivana Jurin, https://orcid.org/0000-0002-2637-9691 • Boris Starčević, https://orcid.org/0000-0002-3090-2772 Igor Rudež, https://orcid.org/0000-0002-7735-6721

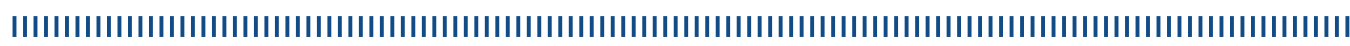

Background: Transcatheter aortic valve implantation (TAVI) was proved to be a preferred method of severe aortic stenosis treatment in patients with medium and high surgical risk. However, that significant superiority over surgery is maintained only if femoral approach is possible. ${ }^{1} \mathrm{Aim}$ : We analyzed changes of approach to TAVI implantation before and after the introduction of percutaneous transfemoral implantation, together with data on 12-months survival, complications and hospital stay.

Results: Among a total of 149 patients who received TAVI in our center, there were 94 implantations before and 55 implantations after the introduction of routine percutaneous implantation when it is feasible (with surgical cut-down reserved only for patients with peripheral artery disease). There were no significant differences in patients' characteristics and risk factors among patients operated during two different periods. All 82 femoral procedures during the first period were performed with surgical cut-down, in addition to 9 transapical and 2 transaortic implantations. In the second period, there were 54 femoral implantations (30 with surgical cut down and 24 full percutaneous) and 1 transapical implantation. 12-month mortality (16\% vs 7\%), and median duration of hospitalization (10 vs 8 days) were lower among patients treated in the second period. However, there were more vascular complications in the second period ( $2 \%$ vs $9 \%$ ), that were mostly resolved during the procedure, or within one day.

Conclusions: After the introduction of full percutaneous approach to TAVI we observed a decrease in postoperative mortality, and reduction of hospital stay length, most probably due to significant decrease in relative proportion of transapical approach and routine surgical cut-down. Relative increase in vascular complications due to persistent selection of transfemoral approach in patients with challenging vascular access did not result in prolonged hospital stay or excess mortality. Future strategies for TAVI, especially during and after the pandemic era, will most probably favor fast-track approach, leaving hospital resources readily and longer available mainly for the most complicated patients.

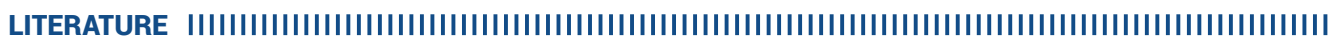

1. Chopra M, Luk NHV, De Backer O, Søndergaard L. Simplification and optimization of transcatheter aortic valve implantation - fast-track course without compromising safety and efficacy. BMC Cardiovasc Disord. 2018 Dec 10;18(1):231. https://doi.org/10.1186/s12872-018-0976-0 